\title{
Stability Analysis for Continuous-Time Switched Systems with Stochastic Switching Signals
}

\author{
Xiaotai Wu, Yang Tang, Jinde Cao, and Xuerong Mao
}

\begin{abstract}
This paper is concerned with the stability problem of randomly switched systems. By using the probability analysis method, the almost surely globally asymptotical stability and almost surely exponential stability are investigated for switched systems with semi-Markovian switching, Markovian switching and renewal process switching signals, respectively. Two examples are presented to demonstrate the effectiveness of the proposed results, in which an example of consensus of multi-agent systems with nonlinear dynamics is taken into account.
\end{abstract}

Index Terms-Switched systems, stability, semi-Markovian switching, Markovian switching, renewal process.

\section{INTRODUCTION}

Randomly switched systems are considered to be a class of switched systems, which consist of a family of distinct active subsystems under operation governed by a stochastic process. According to one randomly switching rule, a subsystem will be activated along the system trajectory at certain instants. The randomly switching usually arises when modeling dynamical systems, which exhibit switching phenomena among several subsystems due to jumping parameters or changing environmental factors. In addition, the randomly switching system can be used to model the system affected by random structural changes. It is known that the randomly switched system serves as a suitable modelling paradigm and has wide applications to diverse areas such as economic systems, manufacturing systems, communication systems and biological systems affected by random delays and component failures [1-5].

Stability analysis of randomly switched systems is a fundamental and challenging research issue. In [2], the multiple Lyapunov-like function method was firstly introduced to investigate the globally asymptotically stability in almost sure sense

This research was partially supported by the National Key R\&D Program of China (2016YFB0303401), the Natural Science Foundation of China (No. 11401005, No. 61403141 and No. 61673176), the Anhui Provincial Promotion Plan Project (No. TSKJ2015B26), the China Postdoctoral Science Foundation (No. 2015M571629), the Anhui Provincial Natural Science Foundation(No. 1608085MA02 and No. 1708085QA16), the Programme of Introducing Talents of Discipline to Universities (the 111 Project under No. B17017) and the Thousand Youth Talents Plan of China. (Corresponding Author: Yang Tang).

$\mathrm{X}$. Wu is with the Department of Mathematics, Southeast University, Nanjing 210096, China, and also with the School of Mathematics and Physics, Anhui Polytechnic University, Wuhu 241000, China. E-mail: (aaxtwu@126.com).

Y. Tang is with the Key Laboratory of Advanced Control and Optimization for Chemical Processes, Ministry of Education, East China University of Science and Technology, Shanghai 200237, China. E-mail: (tangtany@gmail.com).

J. Cao is with the Department of Mathematics, Southeast University, Nanjing 210096, China. E-mail: (jdcao@seu.edu.cn).

$\mathrm{X}$. Mao is with the Department of Mathematics and Statistics, University of Strathclyde, Glasgow, G1 1XH, UK. E-mail: (x.mao@strath.ac.uk).
(GAS a. s.) for randomly switched systems, and stability criteria were also presented for switched systems with Markovian switching. Subsequently, the multiple Lyapunov-like function method has been widely applied to the study on the stability of randomly switched systems [6-9]. A system with Markovian switching is an important randomly switched system, its active time for each subsystem satisfies the exponential distribution, which has limited the applications of Markovian switching. To overcome this restriction, the stability of the system with semi-Markovian switching has been investigated in $[6,9-14]$, where the semi-Markovian switching does not necessarily satisfy memoryless property of Markov switching. In [12], the stability and stabilization were considered for switched systems with discrete-time semi-Markovian signals by using the semi-Markovian kernel approach. The necessary and sufficient conditions for mean square stability are derived for switched systems with semi-Markovian switching signals in [14]. The distribution of sojourn time can depend on both current and next system mode in $[12,14]$.

Due to the failure of a component or abrupt disturbances, a subsystem of a switched system might be unstable. Thus, it is more practical and interesting to consider switched systems with both stable and unstable subsystems [15-18], in which the active time for stable subsystems should be ensured to exceed a threshold such that unstable subsystems can be compensated by stable subsystems. For randomly switched systems with both stable and unstable subsystems, the GAS a. s. was considered for randomly switched systems with different switching signals in [6], where the sojourn time for each subsystem is assumed to satisfy exponential and uniform distributions, respectively. In addition, the GAS a. s. was also examined in [6] for system with a special semi-Markovian switching. It is well known that the stationary distribution of Markovian chains is related to the active time for each state of Markov chains, which thus can be used to restrict the active time of stable and unstable subsystems for system with Markovian switching. Recently, by using the comparison theorem and the stationary distribution of Markov chains, the moment stability was studied for the system with Markovian switching in [8]. In [9], the comparison method introduced in [8] was generalized to investigate the moment stability for systems with semi-Markovian switching.

However, the moment stability presented in [8, 9] can not imply the GAS a. s. and almost surely exponentially stable (ES a. s.), which was also pointed out in [8], and the method provided in $[8,9]$ cannot be directly applied to the investigation on GAS a. s. and ES a. s. Thus, it is still open to address the GAS a. s. and ES a. s. for the system with 
Markovian/semi-Markovian switching by using the stationary distribution of Markov chains/embedded Markov chain. In addition, the sojourn time for switching signals were assumed to satisfy an exponential or a uniform distribution in [6], which are the special cases of renewal process. Hence, the other motivation of this paper is to consider the GAS a. s. and ES a. s. for the system with renewal process switching signals.

Motivated by above discussions, in this paper, we aim to investigate the GAS a. s. and ES a. s. for randomly switched systems, where the switching signal is assumed to satisfy semiMarkovian process, Markovian process and renewal process, respectively. The contributions of this paper are summarized as follows: 1) The GAS a. s. and ES a. s. are investigated for system with Markovian and semi-Markovian switching by using the stationary distribution of the Markov and embedded Markov chain, respectively. In addition, GAS a. s. and ES a. s. are also taken into account for system with renewal process switching signals; 2) A new analysis technique is introduced here to comprehensively consider the GAS a. s. and ES a. s. of randomly switched systems by using the strong law of large number.

Notations: Let $\mathbb{R}=(-\infty,+\infty), \mathbb{R}^{+}=[0,+\infty), \mathbb{N}^{+}=$ $1,2, \cdots, \mathbb{N}=0 \cup \mathbb{N}^{+}$, and $\Gamma=\{1,2, \cdots, r\}$, where $r>0$ is a finite integer. Let $(\Omega, \mathcal{F}, P)$ be a complete probability space with some filtration $\left\{\mathcal{F}_{t}\right\}_{t \geq 0}$ satisfying the usual conditions (i.e., the filtration is increasing and right continuous while $\mathcal{F}_{t_{0}}$ contains all $P$-null sets). Let $\mathcal{C}^{1}$ denote the family of all nonnegative functions $V(x, i)$ on $\mathbb{R}^{d} \times \Gamma \rightarrow \mathbb{R}^{+}$and that are once differentiable in $x$. For $x \in \mathbb{R}^{d},|x|$ denotes the Euclidean norm of $x$. Moreover, let $\mathcal{K}$ denote the class of continuous strictly increasing function $\kappa: \mathbb{R}^{+} \rightarrow \mathbb{R}^{+}$with $\kappa(0)=0$, and $\mathcal{K}_{\infty}$ be the subset of $\mathcal{K}$ functions that are unbounded. $\mathbf{E}$ denotes the expectation operator.

\section{Preliminaries}

In this paper, we consider the following randomly switched system:

$$
\dot{x}(t)=f_{\sigma(t)}(x(t)), t \geq 0,
$$

where $x(t) \in \mathbb{R}^{d}$; the randomly switching signal $\sigma(t) \in \Gamma$ is a piecewise constant function, which is continuous from the right, specifying the index of the active subsystem, i. e., $\sigma(t)=$ $i_{n} \in \Gamma$ for $t \in\left[\tau_{n}, \tau_{n+1}\right)$, and $\tau_{n}$ is the $n$th switching time instant, where $n \in \mathbb{N}$ and $\tau_{0}=0$; the functions $f_{i}: \mathbb{R}^{d} \rightarrow \mathbb{R}^{d}$ are locally Lipschitz and $f_{i}(0)=0$ for $i \in \Gamma$; the probability space $(\Omega, \mathcal{F}, P)$ is generated by the càdlág random process $\sigma(t)$. In addition, it is assumed that there are no jumps in the state $x(t)$ at switching instants.

Let $N(t)$ be the occurrence number for total switching over the interval $[0, t], N_{i}(t)$ be the occurrence number for the $i$ th subsystem over the interval $[0, t]$ and $T_{i}(t)$ be the total time for the switched system active on the $i$ th subsystem over the interval $[0, t]$. Let $s(n)=\tau_{n}-\tau_{n-1}$ be the sojourn time of a subsystem for the $n$th visiting of $\sigma(t)$ and the $s_{i}(n)$ be the sojourn time of the state $i$ for the $n$th visiting of $\sigma(t)$, where $n \in \mathbb{N}^{+}$.

Before giving the main results, we firstly present the following definitions.
Definition 1: $[19,20]$ The switching signal $\sigma(t)$ is said to be

1) semi-Markovian switching, if let $\sigma_{n}=\sigma(t)$ for $\tau_{n} \leq t<$ $\tau_{n+1}, n \in \mathbb{N}$ be the embedded chain,

(i) the discrete-time process $\left\{\sigma_{n}, s(n)\right\}$ is a Markovian renewal process and satisfies

$$
\begin{aligned}
& P\left(\sigma_{n}=i, s(n) \leq t \mid \sigma_{n-1}, s(n-1), \cdots, \sigma_{1}, s(1)\right) \\
= & P\left(\sigma_{n}=i, s(n) \leq t \mid \sigma_{n-1}\right), i \in \Gamma, t \geq 0,
\end{aligned}
$$

(ii) the distribution function of sojourn time $s(n)$ is defined by

$$
F_{i j}(t)=P\left(s(n) \leq t \mid \sigma_{n}=j, \sigma_{n-1}=i\right), i, j \in \Gamma, t \geq 0,
$$

which depends on $\sigma_{n}$ and $\sigma_{n-1}$;

2) Markovian switching, if for $\forall i_{n} \in \Gamma, n \in \mathbb{N}$,

$$
\begin{aligned}
& P\left(\sigma\left(t_{n}\right)=i_{n} \mid \sigma\left(t_{n-1}\right)=i_{n-1}, \cdots, \sigma\left(t_{1}\right)=i_{1}\right) \\
= & P\left(\sigma\left(t_{n}\right)=i_{n} \mid \sigma\left(t_{n-1}\right)=i_{n-1}\right),
\end{aligned}
$$

where $0 \leq t_{1} \leq t_{2} \leq \cdots \leq t_{n}$;

3 ) renewal process switching, if the occurrence $N(t)$ of the switched signal $\sigma(t)$ satisfies a renewal process, that is, the sojourn times $\{s(n), n \in \mathbb{N}\}$ are a sequence of nonnegative independent random variables with a common distribution $F$.

Definition 2: $[2,21]$ The randomly switched system in (1) is said to be

1) globally asymptotically stable almost surely (GAS a. s.), if the following two properties hold simultaneously:

SP1) for any $\varepsilon \in(0,1)$, there is a $\delta=\delta(\varepsilon)>0$ such that such that when $\left|x_{0}\right|<\delta$,

$$
P\left(\sup _{t \geq 0}|x(t)|<\varepsilon\right)>1-\varepsilon,
$$

SP2) for any $h>0$ and $\varepsilon^{\prime}>0$, there is a positive random variable $T\left(h, \varepsilon^{\prime}\right)$ such that $P\left(\sup _{t \geq T\left(h, \varepsilon^{\prime}\right)}|x(t)|<\varepsilon^{\prime}\right)=1$, provided $\left|x_{0}\right|<h$;

2) almost surely exponentially stable (ES a. s.), if

$$
\limsup _{t \rightarrow \infty} \frac{1}{t} \log \left|x\left(t ; x_{0}\right)\right|<0 \text {, a. s., }
$$

for all $x_{0} \in \mathbb{R}^{d}$.

\section{MAIN RESUltS}

In this section, by using the probability analysis method and the strong law of large number, GAS a. s. and ES a. s. will be considered for system (1) with semi-Markovian switching, Markovian switching and renewal process switching signals, respectively.

\section{A. Stability of system with semi-Markovian switching}

In the subsection, the switching signal $\sigma(t)$ is assumed to be governed by a semi-Markovian chain. It can be got from (2) that the embedded chain $\left\{\sigma_{n}, n \in \mathbb{N}\right\}$ is a Markov chain. Let the embedded chain have a transition probability matrix $P=\left[p_{i j}\right]_{r \times r}$, where $p_{i i}=0$ for $i \in r$, and assume that it is irreducible and has a stationary distribution $\bar{\pi}=\left\{\bar{\pi}_{1}, \bar{\pi}_{2}, \cdots, \bar{\pi}_{r}\right\}$. 
Firstly, a lemma is provided to connect the active time on each subsystem and the stationary distribution of the embedded Markov chain, which is crucial to examine the stability of system (1) with semi-Markovian switching.

Lemma 1: Let $\sigma(t)$ be a semi-Markovian process with state sojourn time sequence $s(n), n \in \mathbb{N}$, and $\bar{\pi}$ be the stationary distribution of the embedded Markov chain $\sigma_{n}, n \in \mathbb{N}$. Then for $\forall i \in \Gamma$

$$
\lim _{t \rightarrow \infty} \frac{T_{i}(t)}{t}=\pi_{i}, \quad \text { a. s. }
$$

where

$$
\pi_{i}=\frac{\bar{\pi}_{i} m_{i}}{\sum_{j \in \Gamma} \bar{\pi}_{j} m_{j}},
$$

and $\mathbf{E} s_{i}(1)=m_{i}$.

Proof: A brief proof has been provided in Appendix.

In the following, the GAS a. s. will be presented for system (1) with semi-Markovian switching.

Theorem 1: Assume that there exist functions $\kappa_{1}, \kappa_{2} \in$ $\mathcal{K}_{\infty}$, constants $\mu_{i}>1$ and $\lambda_{i} \in \mathbb{R}, i \in \Gamma$ such that

$\left(\mathrm{H}_{1}\right) \kappa_{1}(|x|) \leq V(x, i) \leq \kappa_{2}(|x|)$,

$\left(\mathrm{H}_{2}\right)$ for $V \in \mathcal{C}^{1}$ and $\sigma(t)=i, i \in \Gamma$,

$$
\frac{\partial V(x, i)}{\partial x} f_{i}(x) \leq \lambda_{i} V(x, i),
$$

$\left(\mathrm{H}_{3}\right) V(x, i) \leq \mu_{i} V(x, j), \forall i, j \in \Gamma$,

$\left(\mathrm{H}_{4}\right) \sum_{i \in \Gamma} \pi_{i}\left(\lambda_{i}+\frac{\ln \mu_{i}}{m_{i}}\right)<0$.

Then system (1) with semi-Markovian switching is GAS a. s.

Proof: It can be derived from $\left(\mathrm{H}_{2}\right)$ and $\left(\mathrm{H}_{3}\right)$ that for $\forall t \in$ $\left[\tau_{n+1}, \tau_{n+2}\right)$ and $\sigma(t)=i_{n+1}, n \in \mathbb{N}$,

$$
\begin{aligned}
& V\left(x(t), i_{n+1}\right) e^{-\lambda_{i_{n+1}}\left(t-\tau_{n+1}\right)} \\
= & V\left(x\left(\tau_{n+1}\right), i_{n+1}\right)+\int_{\tau_{n+1}}^{t} d\left(V\left(x(s), i_{n+1}\right) e^{-\lambda_{i_{n+1}}\left(s-\tau_{n+1}\right)}\right) \\
\leq & V\left(x\left(\tau_{n+1}\right), i_{n+1}\right) \\
\leq & \mu_{i_{n+1}} V\left(x\left(\tau_{n+1}\right), i_{n}\right)
\end{aligned}
$$

which yields that for $\forall t \geq 0$

$$
\begin{aligned}
V(x(t), \sigma(t)) & \leq V\left(x_{0}, i_{0}\right) \prod_{k=1}^{N(t)} \mu_{i_{k}} e^{\int_{0}^{t} \lambda_{\sigma(s)} d s} \\
& =V\left(x_{0}, i_{0}\right) \prod_{i=1}^{r} \mu_{i}^{N_{i}(t)} e^{\int_{0}^{t} \lambda_{\sigma(s)} d s},
\end{aligned}
$$

where $x_{0}$ is the initial value of system (1). Combing this with $\left(\mathrm{H}_{1}\right)$ implies that

$$
\kappa_{1}(|x(t)|) \leq \kappa_{2}\left(\left|x_{0}\right|\right) \prod_{i=1}^{r} \mu_{i}^{N_{i}(t)} e^{\int_{0}^{t} \lambda_{\sigma(s)} d s} .
$$

It follows that

$$
|x(t)| \leq \kappa_{1}^{-1}\left(\kappa_{2}\left(\left|x_{0}\right|\right) \prod_{i=1}^{r} \mu_{i}^{N_{i}(t)} e^{\int_{0}^{t} \lambda_{\sigma(s)} d s}\right) .
$$

By $\left(\mathrm{H}_{4}\right)$, we can select a positive constant $\varepsilon_{1}$ such that

$$
\sum_{i \in \Gamma} \pi_{i} \lambda_{i}+\sum_{i \in \Gamma}\left(\frac{\pi_{i}}{m_{i}}+\varepsilon_{1}\right) \ln \mu_{i}<0 .
$$

It can be got from Lemma 1 that for $\forall i \in \Gamma$

$$
\lim _{t \rightarrow \infty} \frac{T_{i}(t)}{t}=\pi_{i}, \quad \text { a. s. }
$$

On the other hand, it can be checked by using (29) that for $\forall i \in \Gamma$,

$$
\lim _{t \rightarrow \infty} \frac{T_{i}(t)}{N_{i}(t)}=\lim _{t \rightarrow \infty} \frac{\sum_{k=1}^{N_{i}(t)} s_{i}(k)}{N_{i}(t)}=m_{i}, \quad \text { a. s. }
$$

Combing (8) with (9) yields that

$$
\lim _{t \rightarrow \infty} \frac{N_{i}(t)}{t}=\frac{\pi_{i}}{m_{i}}, \quad \text { a. s. }
$$

Thus, for $\forall i \in \Gamma$ and the $\varepsilon_{1}$ defined in (7), there exists a positive constant $T\left(\varepsilon_{1}\right)$ such that if $t \geq T\left(\varepsilon_{1}\right)^{1}$, then,

$$
N_{i}(t) \leq\left(\frac{\pi_{i}}{m_{i}}+\varepsilon_{1}\right) t, \quad \text { a. s. }
$$

It follows that for $t \geq T\left(\varepsilon_{1}\right)$,

$$
\begin{aligned}
& \prod_{i=1}^{r} \mu_{i}^{N_{i}(t)} e^{\int_{0}^{t} \lambda_{\sigma(s)} d s} \\
= & e^{\sum_{i \in \Gamma} N_{i}(t) \ln \mu_{i}} e^{\int_{0}^{t} \lambda_{\sigma(s)} d s} \\
\leq & e^{\left(\sum_{i \in \Gamma}\left(\frac{\pi_{i}}{m_{i}}+\varepsilon_{1}\right) \ln \mu_{i}\right) t} e^{\int_{0}^{t} \lambda_{\sigma(s)} d s} \\
= & e^{\int_{0}^{t}\left(\lambda_{\sigma(s)}+\sum_{i \in \Gamma}\left(\frac{\pi_{i}}{m_{i}}+\varepsilon_{1}\right) \ln \mu_{i}\right) d s}, \quad \text { a.s. }
\end{aligned}
$$

It can be obtained from Lemma 1 that

$$
\begin{aligned}
& \lim _{t \rightarrow \infty} \frac{1}{t} \int_{0}^{t} \lambda_{\sigma(s)} d s \\
= & \lim _{t \rightarrow \infty} \frac{1}{t} \sum_{i \in \Gamma} \int_{0}^{t} \lambda_{i} I_{(\sigma(s)=i)} d s \\
= & \sum_{i \in \Gamma} \lambda_{i} \lim _{t \rightarrow \infty} \frac{T_{i}(t)}{t}=\sum_{i \in \Gamma} \lambda_{i} \pi_{i}, \quad \text { a. s. }
\end{aligned}
$$

Combing this with (7) follows that

$$
\begin{aligned}
& \lim _{t \rightarrow \infty} \frac{1}{t} \int_{0}^{t}\left(\lambda_{\sigma(s)}+\sum_{i \in \Gamma}\left(\frac{\pi_{i}}{m_{i}}+\varepsilon_{1}\right) \ln \mu_{i}\right) d s \\
= & \sum_{i \in \Gamma} \lambda_{i} \pi_{i}+\sum_{i \in \Gamma}\left(\frac{\pi_{i}}{m_{i}}+\varepsilon_{1}\right) \ln \mu_{i}<0, \quad \text { a. s. }
\end{aligned}
$$

which implies that

$$
\lim _{t \rightarrow \infty} \int_{0}^{t}\left(\lambda_{\sigma(s)}+\sum_{i \in \Gamma}\left(\frac{\pi_{i}}{m_{i}}+\varepsilon_{1}\right) \ln \mu_{i}\right) d s=-\infty, \quad \text { a. s. }
$$

Thus, it can be checked by (11) and (13) that

$$
\lim _{t \rightarrow \infty} \prod_{i=1}^{r} \mu_{i}^{N_{i}(t)} e^{\int_{0}^{t} \lambda_{\sigma(s)} d s}=0, \quad \text { a. s. }
$$

To show that system (1) with the semi-Markovian switching is GAS a.s., we first let $h$ and $\varepsilon^{\prime}$ be two arbitrary positive

${ }^{1}$ For almost all $\omega \in \Omega$, there exists a corresponding positive constant $T\left(\varepsilon_{1}, \omega\right)$ (relying on the $\omega$ and $\varepsilon_{1}$ ) such that if $t \geq T\left(\varepsilon_{1}, \omega\right)$, inequality (10) holds. For the convenience, $\omega$ is always omitted in the following. 
numbers. It follows from (14) that there is a positive random variable $T\left(h, \varepsilon^{\prime}\right)$ such that

$$
P\left(\sup _{t \geq T\left(h, \varepsilon^{\prime}\right)}\left(\prod_{i=1}^{r} \mu_{i}^{N_{i}(t)} e^{\int_{0}^{t} \lambda_{\sigma(s)} d s}\right)<\frac{\kappa_{1}\left(\varepsilon^{\prime}\right)}{\kappa_{2}(h)}\right)=1 .
$$

This, together with (6), implies

$$
P\left(\sup _{t \geq T\left(h, \varepsilon^{\prime}\right)}|x(t)|<\varepsilon^{\prime}\right)=1, \quad \forall\left|x_{0}\right|<h .
$$

In other words, SP2) in Definition 2 is fulfilled. Let us now fix any $\varepsilon \in(0,1)$. It follows from (14) that

$$
\sup _{t \geq 0}\left(\prod_{i=1}^{r} \mu_{i}^{N_{i}(t)} e^{\int_{0}^{t} \lambda_{\sigma(s)} d s}\right)<\infty, \quad \text { a. s. }
$$

Hence, there is a positive number $\rho$ such that

$$
P\left(\sup _{t \geq 0}\left(\prod_{i=1}^{r} \mu_{i}^{N_{i}(t)} e^{\int_{0}^{t} \lambda_{\sigma(s)} d s}\right)<\rho\right)>1-\varepsilon .
$$

Let $\delta=\delta(\varepsilon)$ be so small that $\kappa_{2}(\delta)<\kappa_{1}(\varepsilon) / \rho$. Using (6), we therefore see that

$$
P\left(\sup _{t \geq 0}|x(t)|<\varepsilon\right)>1-\varepsilon, \quad \forall\left|x_{0}\right|<\delta .
$$

That is, SP1) in Definition 2 is satisfied too. The proof is therefore complete.

Remark 1: From the definition of semi-Markovian switching, the distribution $F_{i j}(t)$ should depend on $i$ and $j$. Under this assumption, the stability and stabilization problems were investigated in $[12,14]$ for systems with discrete-time semi-Markovian switching by using the semi-Markovian kernel approach. However, for continuous-time semi-Markovian switching, some special assumptions should be given for semiMarkovian process in [6, 9-11]. For example, the distribution $F_{i j}(t)$ only depends on $i$ in [6, 9-11], and satisfies the Phasetype distribution in $[10,11]$. These restrictive assumptions are removed from this paper. Thus, the semi-Markovian signal considered in this paper is more general than in $[6,9-11]$.

As a corollary, the ES a. s. will be given for system (1) with semi-Markovian switching.

Corollary 1: Assume that there exist positive constants $\mu_{i}>1, c$ and $\lambda_{i} \in \mathbb{R}, i \in \Gamma$ such that

$\left(\mathrm{H}_{1}\right) V(x, i) \geq c|x|^{p}$,

$\left(\mathrm{H}_{2}\right)$ for $V \in \mathcal{C}^{1}$ and $\sigma(t)=i, i \in \Gamma$,

$$
\frac{\partial V(x, i)}{\partial x} f_{i}(x) \leq \lambda_{i} V(x, i),
$$

$\left(\mathrm{H}_{3}\right) V(x, i) \leq \mu_{i} V(x, j), \forall i, j \in \Gamma$.

Then,

$$
\limsup _{t \rightarrow \infty} \frac{1}{t} \log \left(\left|x\left(t ; x_{0}\right)\right|\right) \leq \sum_{i \in \Gamma} \pi_{i}\left(\lambda_{i}+\frac{\ln \mu_{i}}{m_{i}}\right), \quad \text { a. s. }
$$

In particular, if $\left(\mathrm{H}_{4}\right)$ in Theorem 1 holds, then system (1) with semi-Markovian switching is ES a. s.

Proof: Combining (5) with (11) yields that, for $\forall i \in \Gamma$ and $\varepsilon_{1}$ defined in (7), there exists a corresponding constant $T\left(\varepsilon_{1}\right)$ such that for $t \geq T\left(\varepsilon_{1}\right)$,

$$
\begin{aligned}
& \log (V(x(t), \sigma(t))) \\
\leq & \log \left(V\left(x_{0}, i_{0}\right)\right)+\sum_{i \in \Gamma} N_{i}(t) \ln \mu_{i}+\int_{0}^{t} \lambda_{\sigma(s)} d s \\
\leq & \log \left(V\left(x_{0}, i_{0}\right)\right)+\left(\sum_{i \in \Gamma}\left(\frac{\pi_{i}}{m_{i}}+\varepsilon_{1}\right) \ln \mu_{i}\right) t+\int_{0}^{t} \lambda_{\sigma(s)} d s, \text { a. s. }
\end{aligned}
$$

It can be checked by using $\left(\mathrm{H}_{1}\right)$ and (12) that

$$
\begin{aligned}
& \limsup _{t \rightarrow \infty} \frac{1}{t} \log \left(\left|x\left(t ; x_{0}\right)\right|\right) \\
\leq & \limsup _{t \rightarrow \infty} \frac{1}{t} \log (V(x(t), \sigma(t))) \\
\leq & \sum_{i \in \Gamma}\left(\frac{\pi_{i}}{m_{i}}+\varepsilon_{1}\right) \ln \mu_{i}+\limsup _{t \rightarrow \infty} \frac{1}{t} \int_{0}^{t} \lambda_{\sigma(s)} d s \\
= & \sum_{i \in \Gamma} \lambda_{i} \pi_{i}+\sum_{i \in \Gamma}\left(\frac{\pi_{i}}{m_{i}}+\varepsilon_{1}\right) \ln \mu_{i}, \quad \text { a. s. }
\end{aligned}
$$

Then, (15) follows by letting $\varepsilon_{1} \rightarrow 0$ in (16). This completes the proof.

\section{B. Stability of system with Markovian switching}

Here, the switching signal $\sigma(t)$ is assumed to be a Markov chain, the GAS a. s. and ES a. s. will be obtained for system (1) with Markovian switching.

Let $\sigma(t) \in \Gamma, t \geq 0$ be a right-continuous Markov chain with generator $Q=\left[q_{i j}\right]_{r \times r}, i, j \in \Gamma$ given by

$P\{\sigma(t+\Delta)=j \mid \sigma(t)=i\}= \begin{cases}q_{i j} \Delta+o(\Delta), & i \neq j, \\ 1+q_{i j} \Delta+o(\Delta), & i=j,\end{cases}$

where $\Delta>0, \lim _{\Delta \rightarrow 0} \frac{o(\Delta)}{\Delta}=0$ and if $i \neq j, q_{i j} \geq 0$ is the transition rate from $i$ to $j$, while $q_{i i}=-\sum_{j \neq i} q_{i j}$. Assume that the considered Markov chain is irreducible, then the Markov chain has a unique stationary distribution $\pi=\left(\pi_{1}, \pi_{2}, \cdots, \pi_{r}\right)$. Set $q_{i}=\left|q_{i i}\right|, i \in \Gamma$.

Lemma 2: [22] The sojourn times $s_{i}(1), s_{i}(2), \cdots$ are independent exponential random variables with parameter $q_{i}, i \in \Gamma$.

In the following, the GAS a. s. is studied for system (1) with Markovian switching.

Theorem 2: Assume that $\left(\mathrm{H}_{1}\right)-\left(\mathrm{H}_{3}\right)$ in Theorem 1 hold, and $\left(\mathrm{H}_{4}\right) \sum_{i \in \Gamma} \pi_{i}\left(\lambda_{i}+q_{i} \ln \mu_{i}\right)<0$, a. s.

Then system (1) with Markovian switching is GAS a. s.

Proof: It can be got from Ergodic theorem [22] (Theorem 3.81) that for $\forall i \in \Gamma$,

$$
\lim _{t \rightarrow \infty} \frac{T_{i}(t)}{t}=\pi_{i}, \quad \text { a. s. }
$$

where $\pi_{i}$ is the stationary distribution of the Markov chain $\{\sigma(t), t \geq 0\}$. By the strong law of large number and Lemma 2 , we have for $\forall i \in \Gamma$,

$$
\lim _{t \rightarrow \infty} \frac{T_{i}(t)}{N_{i}(t)}=\lim _{t \rightarrow \infty} \frac{\sum_{k=1}^{N_{i}(t)} s_{i}(k)}{N_{i}(t)}=\mathbf{E} s_{i}(1)=\frac{1}{q_{i}}, \text { a. s. }
$$

Combining (17) with (18) yields that

$$
\lim _{t \rightarrow \infty} \frac{N_{i}(t)}{t}=\pi_{i} q_{i}, \quad \text { a. s. }
$$


Then, this theorem can be proved similarly as the proof given in Theorem 1, here we omit it.

The ES a. s. will be studied for system (1) with Markovian switching in the following corollary.

Corollary 2: Assume that $\left(\mathrm{H}_{1}\right)-\left(\mathrm{H}_{3}\right)$ in Corollary 1 hold. Then,

$$
\limsup _{t \rightarrow \infty} \frac{1}{t} \log \left(\left|x\left(t ; x_{0}\right)\right|\right) \leq \sum_{i \in \Gamma} \pi_{i}\left(\lambda_{i}+q_{i} \ln \mu_{i}\right), \quad \text { a. s. }
$$

In particular, if $\left(\mathrm{H}_{4}\right)$ in Theorem 2 holds, then system (1) with Markovian switching is ES a. s.

Proof: The proof is similarly to the proof presented in Corollary 1 . Here we omit it.

\section{Stability of system with renewal process switching signals}

In this subsection, the switching signal $\sigma(t)$ is assumed to be governed by a renewal process. Let the state sojourn time sequences $s(n), n \in \mathbb{N}$ and $\sigma\left(\tau_{n}\right), n \in \mathbb{N}$ be mutually independent, and $F(0)=P(s(n)=0)<1$ for avoiding trivialities. In addition, we assume that there exists a probability distribution $p_{i} \in(0,1), i \in \Gamma$, such that for $\forall n \in \mathbb{N}$,

$$
P\left(\sigma\left(\tau_{n+1}\right)=i \mid \sigma\left(\tau_{n}\right), \cdots, \sigma\left(\tau_{0}\right)\right)=p_{i} .
$$

The GAS a. s. and ES a. s. will be investigated for system (1) with this renewal process switching signals. Firstly, a lemma is presented for getting the main result.

Lemma 3: [19] Let $N(t)$ be a renewal process, then

$$
\lim _{t \rightarrow \infty} \frac{N(t)}{t}=\frac{1}{\theta}, \quad \text { a. s., }
$$

where $\mathbf{E} s(n)=\theta, n \in \mathbb{N}$ and $\theta$ is a positive constant.

In the following, the GAS a. s. will be considered for system (1) with renewal process switching signals.

Theorem 3: Assume that there exist functions $\kappa_{1}, \kappa_{2} \in$ $\mathcal{K}_{\infty}$, constants $\mu>1$ and $\lambda_{i} \in \mathbb{R}, i \in \Gamma$ such that

$\left(\mathrm{H}_{1}\right) \kappa_{1}(|x|) \leq V(x, i) \leq \kappa_{2}(|x|)$,

$\left(\mathrm{H}_{2}\right)$ for $V \in \mathcal{C}^{1}$ and $\sigma(t)=i, i \in \Gamma$,

$$
\frac{\partial V(x, i)}{\partial x} f_{i}(x) \leq \lambda_{i} V(x, i),
$$

$\left(\mathrm{H}_{3}\right) V(x, i) \leq \mu V(x, j), \forall i, j \in \Gamma$,

$\left(\mathrm{H}_{4}\right) \sum_{i \in \Gamma} \lambda_{i} p_{i}+\frac{\ln \mu}{\theta}<0$.

Then system (1) with renewal process switching signals is GAS a. s.

Proof: By using $\left(\mathrm{H}_{4}\right)$, there exists a positive constant $\varepsilon_{1}$ such that

$$
\sum_{i \in \Gamma} \lambda_{i} p_{i}+\left(\frac{1}{\theta}+\varepsilon_{1}\right) \ln \mu<0 .
$$

By using $\left(\mathrm{H}_{2}\right),\left(\mathrm{H}_{3}\right)$ and (5), we can get that for $\forall t \geq 0$,

$$
V(x(t), \sigma(t)) \leq V\left(x_{0}, i_{0}\right) \mu^{N(t)} e^{\int_{0}^{t} \lambda_{\sigma(s)} d s} .
$$

Then, for $\forall i \in \Gamma$ and $\varepsilon_{1}$ defined in (20), it can be got from Lemma 3 that there exists a positive constant $T\left(\varepsilon_{1}\right)$ such that if $t \geq T\left(\varepsilon_{1}\right)$,

$$
N(t) \leq\left(\frac{1}{\theta}+\varepsilon_{1}\right) t, \quad \text { a. s. }
$$

Combining this with (21) follows that for $t \geq T\left(\varepsilon_{1}\right)$,

$$
V(x(t), \sigma(t)) \leq \kappa_{2}\left(\left|x_{0}\right|\right) e^{\int_{0}^{t}\left(\lambda_{\sigma(s)}+\left(\frac{1}{\theta}+\varepsilon_{1}\right) \ln \mu\right) d s}, \text { a. s. }
$$

By using the strong law of large number, we have for $l=0,1$,

$$
\begin{aligned}
I_{l} & =: \lim _{t \rightarrow \infty} \frac{1}{t} \int_{\tau_{0}}^{\tau_{N(t)+l}} \lambda_{\sigma(s)} d s \\
& =\lim _{t \rightarrow \infty} \frac{N(t)+l}{t} \frac{1}{N(t)+l} \sum_{k=1}^{N(t)+l} \int_{\tau_{k-1}}^{\tau_{k}} \lambda_{\sigma(s)} d s \\
& =\lim _{t \rightarrow \infty} \frac{N(t)+l}{t} \frac{1}{N(t)+l} \sum_{k=1}^{N(t)+l} \lambda_{\sigma\left(\tau_{k-1}\right)} s(k-1) \\
& =\frac{1}{\theta} \mathbf{E}\left(\lambda_{\sigma\left(\tau_{1}\right)} s(1)\right)=\frac{1}{\theta} \mathbf{E} \lambda_{\sigma\left(\tau_{1}\right)} \mathbf{E} s(1)=\mathbf{E} \lambda_{\sigma\left(\tau_{1}\right)}, \text { a. s., }
\end{aligned}
$$

which yields that

$$
\lim _{t \rightarrow \infty} \frac{1}{t} \int_{0}^{t} \lambda_{\sigma(s)} d s=\mathbf{E} \lambda_{\sigma\left(\tau_{1}\right)}=\sum_{i \in \Gamma} \lambda_{i} p_{i}, \quad \text { a. s. }
$$

Consequently,

$$
\begin{aligned}
& \lim _{t \rightarrow \infty} \frac{1}{t} \int_{0}^{t}\left(\lambda_{\sigma(s)}+\left(\frac{1}{\theta}+\varepsilon_{1}\right) \ln \mu\right) d s \\
= & \sum_{i \in \Gamma} \lambda_{i} p_{i}+\left(\frac{1}{\theta}+\varepsilon_{1}\right) \ln \mu<0, \quad \text { a. s. }
\end{aligned}
$$

It follows that

$$
\lim _{t \rightarrow \infty} \int_{0}^{t}\left(\lambda_{\sigma(s)}+\left(\frac{1}{\theta}+\varepsilon_{1}\right) \ln \mu\right) d s=-\infty, \quad \text { a. s. }
$$

Combing this with (22) implies that

$$
\lim _{t \rightarrow \infty} V(x(t), \sigma(t))=0, \quad \text { a. s. }
$$

Then, by utilizing the proof of Theorem 1, we can similarly derive that system (1) with renewal process switching signals is GAS a. s. This completes the proof.

By the proof of Theorem 3, the ES a. s. can be obtained as a corollary.

Corollary 3: Assume that there exist positive constants $\mu>$ $1, c$ and $\lambda_{i} \in \mathbb{R}, i \in \Gamma$ such that

$\left(\mathrm{H}_{1}\right) V(x, i) \geq c|x|^{p}$,

$\left(\mathrm{H}_{2}\right)$ for $V \in \mathcal{C}^{1}$ and $\sigma(t)=i, i \in \Gamma$,

$$
\frac{\partial V(x, i)}{\partial x} f_{i}(x) \leq \lambda_{i} V(x, i),
$$

$\left(\mathrm{H}_{3}\right) V(x, i) \leq \mu V(x, j), \forall i, j \in \Gamma$.

Then,

$$
\limsup _{t \rightarrow \infty} \frac{1}{t} \log \left(\left|x\left(t ; x_{0}\right)\right|\right) \leq \sum_{i \in \Gamma} \lambda_{i} p_{i}+\frac{\ln \mu}{\theta}, \quad \text { a. s. }
$$

In particular, if $\left(\mathrm{H}_{4}\right)$ in Theorem 3 holds, then system (1) with renewal process switching signals is ES a. s.

Proof: The proof is similarly to the proof presented in Corollary 1 . Here we omit it.

Remark 2: It is worth pointing out that condition $\left(\mathrm{H}_{4}\right)$ in Theorem 3 implies that when $\sum_{i \in \Gamma} \lambda_{i} p_{i}<0$, we need $\theta>\frac{-\ln \mu}{\sum_{i \in \Gamma} \lambda_{i} p_{i}}$ to get the GAS a. s. and ES a. s., that is, if 
the expectation of sojourn time $\theta$ is larger than $\frac{-\ln \mu}{\sum_{i \in \Gamma} \lambda_{i} p_{i}}$, the stability can be guaranteed.

Remark 3: Though the GAS a. s. was also considered in $[2,6]$, it is worth pointing out that the system model considered in this paper is more general. In [2], the GAS a. s. was investigated for randomly switched systems with all stable subsystems. In [6], the GAS a. s. was considered for randomly switched systems, where the sojourn time of switched signals satisfies an exponential distribution in Theorem 3.2 and a uniform distribution in Theorem 3.4, respectively. It is well known that the sojourn times $\{s(n), n \in \mathbb{N}\}$ of renewal process are a sequence of nonnegative independent random variables with a common distribution $F$, where the distribution $F$ may satisfy an exponential distribution or a uniform distribution. Thus, the renewal process switching signals considered in Theorem 3 contain switching signals considered in Theorems 3.2 and 3.4 of [6] as a special case.

Remark 4: By using the comparison principle, the moment stability was investigated for the system with Markovian switching in [8] and with semi-Markovian switching in [9], respectively. Unfortunately, the moment stability cannot yield GAS a. s. [23]. In addition, the methods used in [8, 9] cannot be directly applied to investigating the GAS a. s. Thus, a new analysis technique is introduced in this paper to consider the GAS a. s. and ES a. s., which has provided a uniform method to investigate the stability for systems with semi-Markovian switching, Markovian switching and renewal process switching signals, respectively.

\section{EXAMPLES}

In this section, two examples are given to show the effectiveness of the main results. The first example is a switched system with both stable and unstable subsystems, and some comparisons will be given between this paper and some wellstudied existing works. In the second example, it is shown that the results in this paper can be used to consider the consensus of multi-agent systems with semi-Markovian switching and nonlinear dynamics.

Example 1: In this example, the switched systems contains both stable and unstable subsystems. Consider the following dynamic system:

$$
\dot{x}(t)=f_{\sigma(t)}(x(t)),
$$

where

$$
\begin{aligned}
& f_{1}(x)=\left[\begin{array}{c}
-3 x_{1}+x_{2} \\
\left(x_{1}+x_{2}\right) \sin x_{1}-3 x_{2}
\end{array}\right] \\
& f_{2}(x)=\left[\begin{array}{c}
-1.5 x_{1}-x_{1}^{3} \\
x_{1}-0.75 x_{2}
\end{array}\right], f_{3}(x)=\left[\begin{array}{l}
x_{1} \\
x_{2}
\end{array}\right] .
\end{aligned}
$$

In the following, it will be assumed that the switching signal $\sigma(t)$ satisfies Markovian process, a renewal process and a semi-Markovian process, respectively, and shown that system (24) with the assumed switching signal is GAS a. s. and ES a. s. Consider the following three candidate Lyapunov functions

$$
V_{1}(x)=x_{1}^{2}+x_{2}^{2}, V_{2}(x)=\frac{1}{2}\left(x_{1}^{2}+x_{2}^{2}\right), V_{3}(x)=\frac{1}{2} x_{1}^{2}+x_{2}^{2},
$$

which yields that $\lambda_{1}=-3, \lambda_{2}=-1$ and $\lambda_{3}=2$. We can select $\mu_{1}=2, \mu_{2}=1.01$ and $\mu_{3}=2$.

Let the switching signal $\sigma(t)$ be a Markov chain, and the generator of the Markov chain be

$$
Q=\left[\begin{array}{ccc}
-2 & 1 & 1 \\
4 & -4 & 0 \\
2 & 1 & -3
\end{array}\right]
$$

Then, we have the invariant distribution $\pi=(0.6,0.2,0.2)$. It can be checked that $\sum_{i \in \Gamma} \pi_{i}\left(\lambda_{i}+q_{i} \ln \mu_{i}\right)=-0.483<0$. Fig. 1 (a) presents the state trajectories for system (24) with Markovian switching.
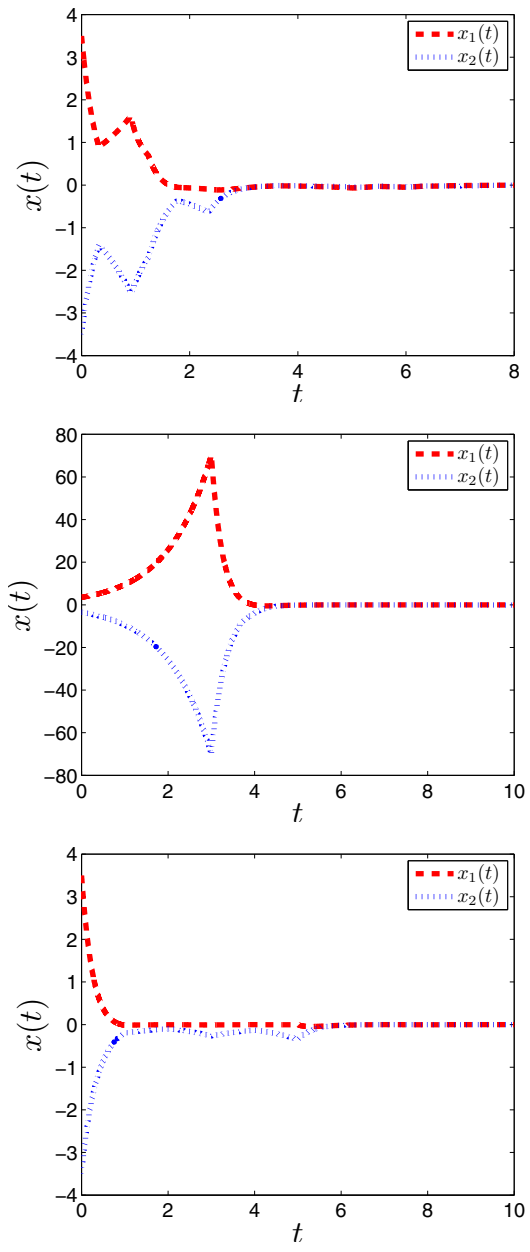

Fig. 1. (a) State trajectories of $x(t)$ for system (24) with Markovian signals. (b) State trajectories of $x(t)$ for system (24) with renewal process signals. (c) State trajectories of $x(t)$ for system (24) with semi-Markovian signals.

If the switching signal $\sigma(t)$ is a renewal process, and $p_{1}=$ $0.6, p_{2}=0.2, p_{3}=0.2$, then we have $\sum_{i \in \Gamma} \lambda_{i} p_{i}+\frac{\ln \mu}{\theta}<0$, when $\theta>0.432$. Fig. 1 (b) shows that when $\theta=1$, system (24) with renew process switching signals is stable.

If the switching signal $\sigma(t)$ is a semi-Markovian process, and the transition probability matrix for the embodied chain is

$$
P=\left[\begin{array}{ccc}
0 & 0.25 & 0.75 \\
0.42 & 0 & 0.58 \\
0.36 & 0.64 & 0
\end{array}\right]
$$


and has a unique stationary distribution

$$
\bar{\pi}=[0.3986,0.3409,0.2605] .
$$

In addition, let the expectations of sojourn time for states 1,2 and 3 be $m_{1}=2, m_{2}=2$ and $m_{3}=3$, respectively. Thus, we get $\pi=[0.3527,0.3016,0.3457]$ by using Lemma 1 . Then, $\sum_{i \in \Gamma} \pi_{i}\left(\lambda_{i}+\frac{\ln \mu_{i}}{m_{i}}\right)=-0.4647<0$. Fig. 1 (c) presents the state trajectories for system (24) with semi-Markovian switching.

In this example, the subsystems of switched systems can be stable and unstable. Ref. [2] cannot be applied to switched systems with unstable subsystems. For renewal process, we give an easy-to-check criterion, where the distribution of sojourn time contains the uniform distribution and exponential distribution in [6] as a special case. In addition, for switched systems with continuous-time semi-Markovian signals, the distribution $F_{i j}$ in this example depends on $i$ and $j$. Thus, Refs. [9-11] have assumed the distribution $F_{i j}$ only depends on $i$, which cannot be used in this example.

Example 2: In the following, A coupled dynamic system was considered in [24, 25]

$$
\frac{d x^{i}(t)}{d t}=f\left(x^{i}(t), t\right)+\sum_{j=1}^{m} a_{i j}^{k} x^{j}(t), t \in\left[\tau_{k-1}, \tau_{k}\right),
$$

where $i=1, \cdots, 5, A_{k}=\left[a_{i j}^{k}\right] \in S_{G}, k \in \mathbb{N}^{+}, S_{G}=$ $\left\{G_{l} \mid G_{l}=\left[g_{i j}^{l}\right]_{i, j=1}^{n} \in R^{n \times n}, l=1,2,3\right\}$. In addition, we assume that each $G_{l}$ is a matrix with zero row sum and nonnegative off diagonal entries. For any $A$, define $R V(A)=$ $\min _{i, j}\left\{a_{i j}+a_{j i}+\sum_{k \neq i, j} \min \left\{a_{i k}, a_{j k}\right\}\right\}$. Then, $R V(G) \mathrm{de}-$ fined by $R V\left(G_{l}\right)=\min _{i, j}\left\{g_{i j}^{l}+g_{j i}^{l}+\sum_{k \neq i, j} \min \left\{g_{i k}^{l}, g_{j k}^{l}\right\}\right\}$ is a random variable on $S_{G}$. In [24], the consensus was considered for system (25) with independent identically distributed (i. i. d.) and Markov chains switching signals, respectively. In the following, it will be shown that system (25) achieves almost sure consensus with semi-Markovian switching signals under our results.

The dynamics of each node is assumed to be a Chua's circuit

$$
f(x, t)=\left\{\begin{array}{l}
p\left[-x_{1}+x_{2}+g\left(x_{1}\right)\right] \\
x_{1}-x_{2}+x_{3} \\
-q x_{2}
\end{array}\right.
$$

where $g\left(x_{1}\right)=m_{0} x_{1}+\frac{1}{2}\left(m_{1}-m_{0}\right)\left(\left|x_{1}+1\right|-\left|x_{1}-1\right|\right)$, $m_{0}=-0.68, m_{1}=-1.27, p=9, q=12.87$.

Let $S_{G}=\left\{G_{1}, G_{2}, G_{3}\right\}$, where the randomly three coupling matrices are defined as in [24],

$$
\begin{aligned}
G_{1} & =\left[\begin{array}{ccccc}
-2.2889 & 0.8464 & 0.9573 & 0.2553 & 0.2299 \\
0.6423 & -2.6972 & 0.6203 & 0.8586 & 0.5761 \\
0.2213 & 0.2789 & -2.2218 & 0.9111 & 0.8106 \\
0.8371 & 0.7466 & 0.1726 & -2.1601 & 0.4038 \\
0.9711 & 0.2369 & 0.0903 & 0.7252 & -2.0235
\end{array}\right], \\
G_{2} & =\left[\begin{array}{ccccc}
-1.4357 & 0.5566 & 0.3178 & 0.2699 & 0.2915 \\
0.3209 & -2.1481 & 0.4522 & 0.5246 & 0.8504 \\
0.5114 & 0.8300 & -3.2257 & 0.9727 & 0.9116 \\
0.0606 & 0.8588 & 0.1099 & -1.6685 & 0.6393 \\
0.7257 & 0.7890 & 0.1097 & 0.3119 & -1.9363
\end{array}\right], \\
G_{3} & =\left[\begin{array}{lllll}
-1.5879 & 0.5846 & 0.3934 & 0.1338 & 0.4761 \\
0.8383 & -3.2444 & 0.8266 & 0.6715 & 0.9081 \\
0.5847 & 0.8277 & -2.5356 & 0.5710 & 0.5522 \\
0.9481 & 0.1910 & 0.2076 & -1.3796 & 0.0329 \\
0.0610 & 0.4425 & 0.3181 & 0.1477 & -0.9693
\end{array}\right] .
\end{aligned}
$$

It has been checked in [24] that $R V\left(G_{1}\right)=$ $1.7835, R V\left(G_{2}\right)=1.2884, R V\left(G_{3}\right)=0.6402$. Assume the transition probability matrix for the embedded chain is

$$
P=\left[\begin{array}{ccc}
0 & 0.8 & 0.2 \\
0.7 & 0 & 0.3 \\
0.6 & 0.4 & 0
\end{array}\right]
$$

and has a unique stationary distribution

$$
\bar{\pi}=[0.4,0.4,0.2] .
$$

In addition, let expectations of sojourn time for states 1,2 and 3 be $m_{1}=1, m_{2}=3$ and $m_{3}=2$, respectively. Thus, we get $\pi=[0.2,0.6,0.2]$.
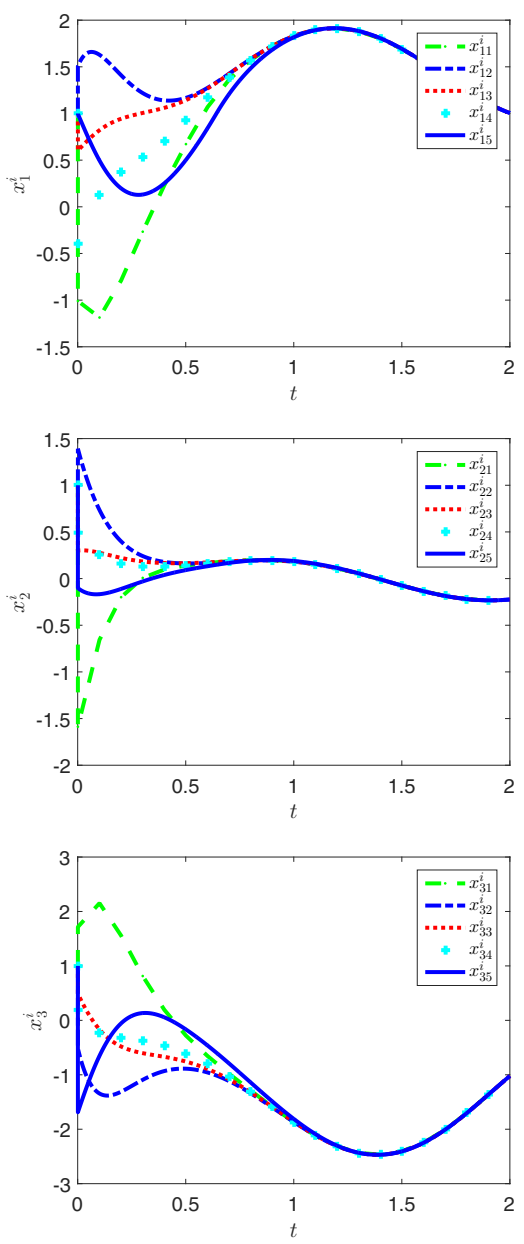

Fig. 2. State trajectories of $x_{1}^{i}(t), x_{2}^{i}(t)$ and $x_{3}^{i}(t)$ for system (25) with semi-Markovian switching.

By utilizing Theorem 1 in [24] and the definition of $R V(G)$, we can get from Theorem 1 in this paper that if $\sum_{i \in \Gamma} \pi_{i}(L-$ $\left.\varepsilon R V\left(G_{i}\right)+\frac{\ln \mu_{i}}{m_{i}}\right)<0$, where $L=12.87$ and $\varepsilon=10.5$ are defined as in [24], then system (25) with semi-Markovian switching is almost sure synchronous. Here, we let $\mu_{i}=1.01$, and get $\sum_{i \in \Gamma} \pi_{i}\left(L-\varepsilon R V\left(G_{i}\right)+\frac{\ln \mu_{i}}{m_{i}}\right)=-0.3317<0$, which implies that system (25) with semi-Markovian switching achieves almost sure consensus. Fig. 2 is the simulation result of $x^{i}(t)$, where the sojourn time sequence $s_{i}(n), n \in \mathbb{N}^{+}$is an i. i. d. sequence and satisfies a uniform distribution on the 
interval $[0,2 l]$ for $l=1,2,3$. It can be seen from Fig. 2 that the system (25) with semi-Markovian switching achieves almost sure consensus.

\section{CONCLUSiOnS}

In this paper, the GAS a. s. and ES a. s. have been considered for the randomly switched systems by using the probability analysis method. Three kinds of switching signals are investigated in this paper including semi-Markovian switching, Markovian switching and renewal process switching signals. Two examples have been presented to illustrate the effectiveness of the proposed results. The sojourn time sequence and embodied chain have assumed to be mutually independent for renewal process, which limits the application of the results. Our future research topic will try to generalize this assumption.

\section{ACKNOWLEDGMENTS}

The authors would like to thank the Associate Editor and anonymous referees for their helpful comments and suggestions which have greatly improved this paper.

\section{APPENDIX}

Proof of Lemma 1: It can be checked that

$$
\begin{aligned}
\frac{T_{i}(t)}{t} & =\frac{\sum_{k=1}^{N_{i}(t)} s_{i}(k)}{\sum_{j \in \Gamma} \sum_{k=1}^{N_{j}(t)} s_{j}(k)} \\
& =\frac{\frac{N_{i}(t)}{N(t)} \frac{1}{N_{i}(t)} \sum_{k=1}^{N_{i}(t)} s_{i}(k)}{\sum_{j \in \Gamma} \frac{N_{j}(t)}{N(t)} \frac{1}{N_{j}(t)} \sum_{k=1}^{N_{j}(t)} s_{j}(k)} .
\end{aligned}
$$

As the embedded Markov chain $\left\{\sigma_{n}, n \in \mathbb{N}\right\}$ is an irreducible Markov chain, by using Ergodic theorem (Theorem 1.10.2 in [22]), we have

$$
\lim _{t \rightarrow \infty} \frac{N_{i}(t)}{N(t)}=\bar{\pi}_{i}, \quad \text { a. s. }
$$

On the other hand, it can be derived from (3) that for $n \in \mathbb{N}$,

$$
\begin{aligned}
P\left(s_{i}(n) \leq t\right) & =P\left(\tau_{n} \leq t \mid \sigma_{n-1}=i\right) \\
& =\sum_{j \in \Gamma} P\left(\tau_{n} \leq t, \sigma_{n}=j \mid \sigma_{n-1}=i\right) \\
& =\sum_{j \in \Gamma} F_{i j}(t) p_{i j},
\end{aligned}
$$

which implies that $s_{i}(n)$ are i. i. d. random variables with $\mathbf{E} s_{i}(n)=m_{i}, n \in \mathbb{N}$. Then, it can be checked by the strong law of large numbers that for $\forall i \in \Gamma$,

$$
\lim _{t \rightarrow \infty} \frac{1}{N_{i}(t)} \sum_{n=1}^{N_{i}(t)} s_{i}(n)=m_{i}, \quad \text { a. s. }
$$

Substituting (29) and (28) into (27) yields that

$$
\lim _{t \rightarrow \infty} \frac{T_{i}(t)}{t}=\frac{\bar{\pi}_{i} m_{i}}{\sum_{j \in \Gamma} \bar{\pi}_{j} m_{j}}, \text { a. s. }
$$

This completes the proof.

\section{REFERENCES}

[1] C. G. Cassandras and J. Lygeros, "Stochastic hybrid systems: Research issues and areas," in Stochastic Hybrid Systems. Boca Raton, FL: CRC Press, 2007, Autom. Control Eng., pp. 1-14, 2007.

[2] D. Chatterjee and D. Liberzon, "On stability of randomly switched nonlinear systems," IEEE Transactions on Automatic Control, vol. 52 , pp. 2390-2394, 2007.

[3] Y. Kang, D. Zhai, G. Liu, Y. Zhao, and P. Zhao, "Stability analysis of a class of hybrid stochastic retarded systems under asynchronous switching," IEEE Transactions on Automatic Control, vol. 59, pp. 15111523, 2014.

[4] X. Yang and J. Lu, "Finite-time synchronization of coupled networks with Markovian topology and impulsive effects," IEEE Transactions on Automatic Control, vol. 61, no. 8, pp. 2256-2261, 2016.

[5] Y. Tang, H. Gao, J. Lu, and J. Kurths, "Pinning distributed synchronization of stochastic dynamical networks: a mixed optimization approach," IEEE Transactions on Neural Networks and Learning Systems, vol. 25, no. 10 , pp. 1804-1815, 2014.

[6] D. Chatterjee and D. Liberzon, "Stabilizing randomly switched systems," SIAM Journal on Control and Optimization, vol. 49, pp. 2008-2031, 2011.

[7] F. Zhu, Z. Han, and J. Zhang, "Stability analysis of stochastic differential equations with Markovian switching," Systems Control Letters, vol. 61, pp. 1209-1214, 2012.

[8] J. Leth, H. Schioler, M. Gholami, and V. Cocquempot, "Stochastic stability of Markovianly switched systems," IEEE Transactions on Automatic Control, vol. 58, no. 8, pp. 2048-2054, 2013.

[9] H. Schioler, M. Simonsen, and J. Leth, "Stochastic stability of systems with semi-Markovian switching," Automatica, vol. 50, pp. 2961-2964, 2014.

[10] Z. Hou, J. Luo, P. Shi, and S. K. Nguang, "Stochastic stability of Ito differential equations with semi-Markovian jump parameters," IEEE Transactions on Automatic Control, vol. 51, pp. 1383-1387, 2006.

[11] F. Li, L. Wu, P. Shi, and C. Lim, "State estimation and sliding mode control for semi-Markovian jump systems with mismatched uncertainties," Automatica, vol. 51, pp. 385-393, 2015.

[12] L. Zhang, Y. Leng, and P. Colaneri, "Stability and stabilization of discrete-time semi-Markov jump linear systems via semi-Markov kernel approach," IEEE Transactions on Automatic Control, vol. 61, pp. 503$508,2016$.

[13] M. Ogura and V. M. Preciado, "Stability of Markov regenerative switched linear systems," Automatica, vol. 69, pp. 169-175, 2016.

[14] L. Zhang, Y. Leng, and P. Colaneri, "Stability and stabilization of semi-Markov jump linear systems with exponential modulated periodic distribution of sojurn time," IEEE Transactions on Automatic Control, vol. 62 , no. 6, pp. 2870-2885, 2017.

[15] G. Zhai, B. Hu, K. Yasuda, and A. N. Michel, "Stability analysis of switched systems with stable and unstable subsystems: An average dwell time approach," International Journal of Systems Science, vol. 32, no. 8 , pp. 1055-1061, 2001.

[16] W. Feng and J. Zhang, "Stability analysis and stabilization control of multi-variable switched stochastic systems," Automatica, vol. 42, no. 1, pp. 169-176, 2006.

[17] L. Zhang and H. Gao, "Asynchronously switched control of switched linear systems with average dwell time," Automatica, vol. 46, no. 5, pp. 953-958, 2010 .

[18] W. Feng, J. Tian, and P. Zhao, "Stability analysis of switched stochastic systems," Automatica, vol. 47, no. 1, pp. 148-157, 2011.

[19] S. M. Ross, Stochastic Processes. John Wiley, Sons, Inc, 1996.

[20] V. S. Barbu and N. Limnios, Semi-Markov Chains and Hidden SemiMarkov Models toward Applications. springer, 2008.

[21] X. Mao, Stochastic Differential Equations and Applications (2nd ed.). Chichester: Howrwood, 2007.

[22] J. Norris, Markov Chains. Cambridge University Press: New York, 2009.

[23] O. Kallenberg, Foundations of Modern Probability (2nd ed.). SpringerVerlag, 2002.

[24] B. Liu, W. Lu, and T. Chen, "Synchronization in complex networks with stochastically switching coupling structures," IEEE Transactions on Automatic Control, vol. 57, no. 3, pp. 754-760, 2012.

[25] X. Wu, Y. Tang, and W. Zhang, "Stability analysis of stochastic delayed systems with an application to multi-agent systems," IEEE Transactions on Automatic Control, vol. 61, no. 12, pp. 4143-4149, 2016. 